\title{
Computer Assisted Alerts Using Mental Model Approach for Customer Service Improvement
}

\author{
Abid Ghaffar ${ }^{1,2}$, Mohamed Ridza Wahiddin ${ }^{1}$, Asadullah Shaikh ${ }^{3}$ \\ ${ }^{1}$ Department of Computer Science, International Islamic University Malaysia, Kuala Lumpur, Malaysia; ${ }^{2}$ Department of Computer \\ Science, Umm Al-Qura University, Makkah, Kingdom of Saudi Arabia; ${ }^{3}$ Department of Information Technology, Shaheed Benazir \\ Bhutto University, Shaheed Benazirabad, Pakistan. \\ Email: aaghaffar@uqu.edu.sa, abid.ghafffar@live.iium.edu.my,mridza@iium.edu.my, shaikhasad@hotmail.com
}

Received 2013

\begin{abstract}
Warning alerts are specially designed to protect user rights and safety to avoid serious damage caused by overlooking the essence of warning alerts. Today's world of Information Communication Technology (ICT) needs improvement and to review the decisions of security experts in terms of improving warning designs and dialogues, and timely inform the authorities to take quick action at the right time and choice. Human behaviour is deeply involved in most of the security failures and its poor response. If we are able to check and monitor human behaviour in any organisation, we can achieve quality assurance and provide best services to our customers. We have arranged a study in the Center of Post Graduate Studies, International Islamic University, Malaysia (CPS-IIUM), department of Hajj Services-Makkah, and Hospital Management System-Makkah comprised of Observation, Interviews, Questionnaire and discussion based on organizational structure and job activities of people involved in different scenarios and positions under one umbrella of organizational objectives in order to trap the human error in order to take rapid action and response from the management team. Human behaviour is deeply observed and checked while performing different job activities in order to identify the serious errors at the right time during job performance at various levels. We have applied the concept of Brahm's Language for the simulation of human behaviour which proves an opportunity to simulate human behaviour while performing job activities. Customer service can be improved easily if necessary measures and decisions are taken at the right time and place in any organisation.
\end{abstract}

Keywords: Mental Model Approach; Warning Dialogues; Warning Alerts; Computer Alerts; Security and Privacy; Cognitive Science

\section{Introduction}

Customer Service Department has many practical challenges to meet the formal requirements, but some issues remained unresolved and hidden for long and do not come up with solution. Consequently, organizational strength and objectives are compromised to a great extent and might result in organizational sufferings or collapse, therefore, it is highly recommended to take rapid action against those shortfalls which are compromising the objectives of an organization and leading us towards failure state.

It has been observed in sensitive departments like military forces, nuclear installations, weapon development industries, hospitals, security related offices, automated transport systems like computerized trains etc. that even a small mistake or an error in day to day affairs in an organization might cause a huge damage or loss not only to an organization but also to the natural environment and people around [7]. The idea is to trap those human errors which can be checked and warning alert could be issued well in time in order to take rapid action at the spot [1].

In order to address the quality assurance in the customer service department we have arranged a research study to monitor the job activities of an individual in order to design mental model which will lead us towards organizational goal and objectives [5].

There is a great challenge to trap the human activity error in order to generate the warning alerts, Brahms Language provides us full opportunity to proceed in this direction. Modeling for the real world practice has been performed to define human activity system, later, real world practice and computational modeling was established [2].

The remainder of the paper provides an overview of the mental model design and generation of warning alert system during the course of error in the work practice system. In Section 2 the strategy which has been used for 
designing mental model and generation of warning codes is discussed. Section 3 presents the related research work in the field of human activities based on work practice system and warning alert system. Section 4 explores the feedback loop which improves existing work practice system. Section 5 focuses on warning code system. In Section 6 the application of Brahms Modeling and Simulation is addressed. Finally, Section 7 provides the conclusion and identifies directions for future work.

\section{Research Methodology}

We have chosen three departments for our mental model design, i.e., Centre of Post Graduate Studies, International Islamic University, Malaysia, Hajj DepartmentMakkah, Saudi Arabia, Hospital Department for Patient Records, Saudi Arabia. Currently, we are working on CPS-IIUM Mental Model Design and we have successfully designed the human behavioural activities for one user as given below. We have conducted interviews/ questionnaire from different employees working in the CPS-IIUM and tried to monitor the job activities. The major objective of job for each position of an employee is kept in mind and we have observed all the steps while performing job activities of an employee [8]. We have applied quantitative and qualitative research methodology.

Figure 1 represents the mental model design for CPS-IIUM (customer service) is being presented which starts from observation and reaches towards potential results through judgment and action plan. Warning alert would be generated if any error occurred in the job activity of an individual [1]. The whole process involved Observation, Judgment, Action and Results. It clearly indicates how we can achieve quality results if proper job patterns are followed and obliged [5].

Different warning codes have been defined based on the sensitivity of errors and it is activated whenever error is generated in certain situation as a result of human behavioural activity in the system [1,3,4]. In case of CPS-IIUM modeling system, we have observed different activity level e.g. Documents Received, Lack of IT Support, Lack of Coordination with staff members, Lack of Resources, Lack of Appreciation, Health Issues, Absence from Duty, Incompatible Rules, Lack of Skills, Overburdened, Personal Problems etc. In the second phase called "Judge and Decide" (Figure 1), decisions are taken like Documents Evaluation, Request for Resources, Learn Skills, Rule Update, Discussion on Issues, Adopt healthy habits and Goal Settings etc. Similarly, in the third phase called "Act" which consists of action part after completing judgment and decision phases, it contains Decision Taken, Acquire better Resources, Maximize Availability, Active Role Playing, Rules Followed, Timely Decision, Weak Decision Making, Breaking Rules, Inefficient and Documents sent to Record Unit etc. Consequently, the potential results are achieved in terms of Quality Assurance. We achieve two types of results either positive or negative depending on the job pattern followed by the employee in the department which is under consideration for mental model design. Quick replies to emails, phone calls, postal mails and customer status is always known if proper job pattern is followed as mentioned in the mental model for the customer service. On the other hand, potential results in the form of late replies to emails, phone calls and postal mails, moreover, customer status is always unknown if improper job pattern is followed [6].

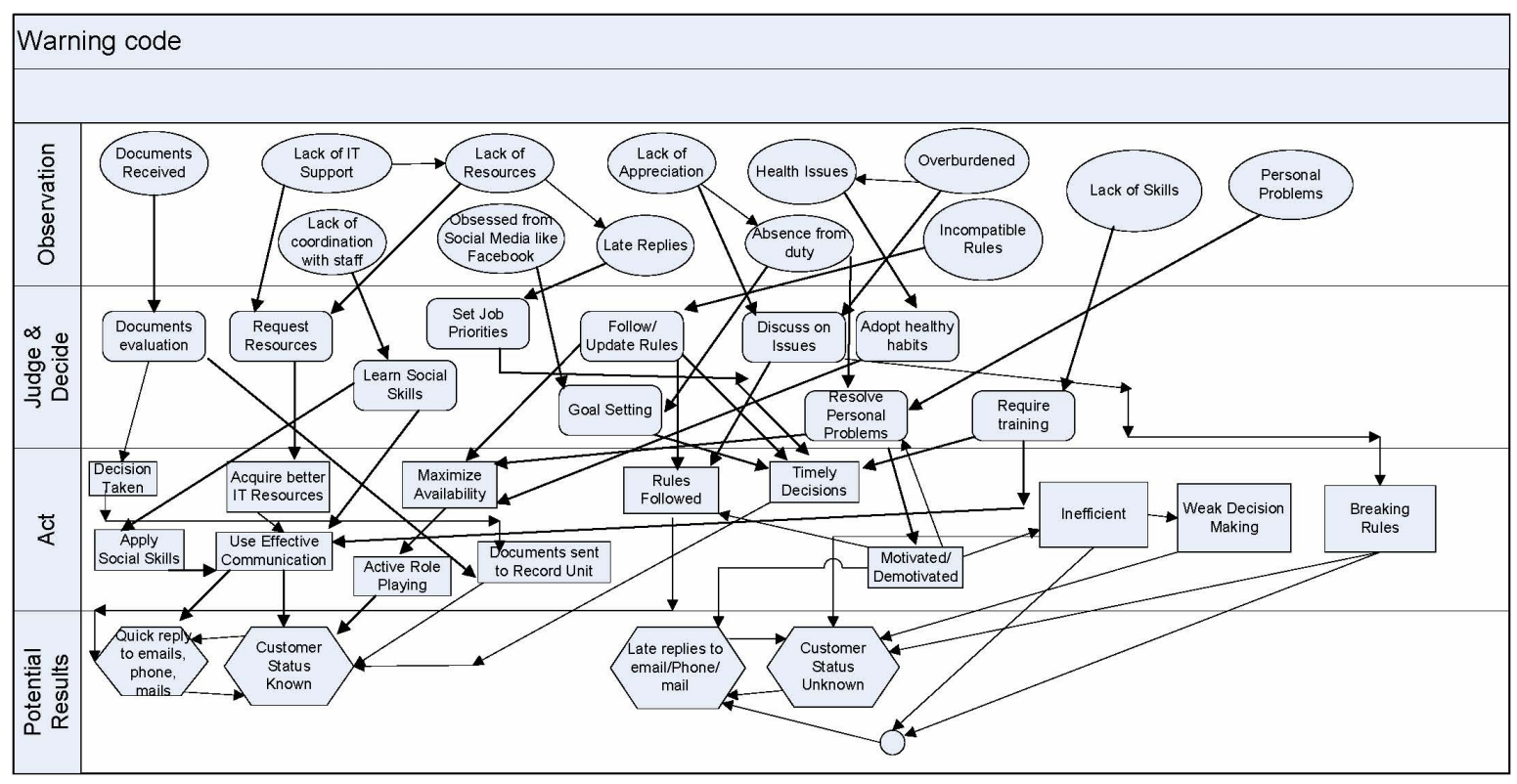

Figure 1. Mental model design for customer service improvement [1]. 
It is a great challenge for observers to record human behaviour in certain situations as human behaviour changes with respect to time and mood [5], therefore, customer feed-back is also recorded and observed directly which leads us to design mental model of CPSIIUM. During the whole process of observation, judgement, and action part, it has been keenly observed that information must be recorded with the help of interviews and questionnaire. All the questions laid down based on the direct observation and based on the organisational goals.

Example of research questions are:

Q1. What is your job title?

Q2. What are your job activities?

Q3. Are you satisfied with your job objectives?

Q4. How many times you take personal leave in a year?

Q5. How much time you spend in social media in a day?

\section{Related Work}

Most of the security applications have loop holes in their implementation part just due to the irresponsible human role and behaviour while dealing with security issues [9].

Geong Sen Poh et al. (2012), presented an idea about HBSF called Human Behaviour Security Framework while dealing with security related issues [8]. He has designed some security framework where we can provide safety to the system [8].

Jim Blythe et al. (2011), presented an idea about simulation of human behaviour in testing cyber security. It reflects the human role in the security framework [9]. There are possibilities to simulate different security be behaviour but at some point we have to rely on human behaviour role which have been experienced a security risk [9].

Cristian Bravo-Lillo et al. (2011), gave an idea about designing warning dialogues and messages which may lead to safe behaviour. She focused on the basic concept of designing warning dialogues and messages at proper location while designing software applications [1].

Maarten Sierhuis et al. (2002), gave an idea for work system design with the help of Brahm's Model. He focused on the observation of human behaviour while performing job in the real work practice system, and with the help of Brahm's Simulation and Modeling technique work practice system can be designed [2].

In 2011, Lorrie Faith Cranor presented an idea about human framework while dealing with security warnings. There are different factors involved in human behaviour e.g. psychological, emotional, knowledge and motivation to take a certain decision [5]. Similarly, Jim Blythe USC et al. (2011) proposed the concept of risk communication while displaying warning dialogues and alerts [6].
Nevertheless, existing research is focused on the mutual relationship between human behaviour and security technologies. It doesn't give us a way out plan or model a work practice system which can detect its own errors either caused by human behaviour or by security technologies.

\subsection{Our Contribution}

Our main objective is to design such a model which can generate warning alerts in a real work practice system by using the concept of Brahm's Modeling and Simulation [2]. Human behaviour is deeply involved while performing different job activities in an organisation, it's quite difficult to trap the human error in the real working environment where so many factors are involved to determine the efficiency of an individual.

Our working model generates the warning alerts which could lead the organisation from failure state to the successful state. Moreover organisational targets and goals can be easily met if it is applied in its letter and spirit. Consequently, organizational quality and efficiency can be improved and maximized, quality assurance can be guaranteed.

\section{Feedback Loop in the Work Practice System}

Figure 2 shows the input in the work practice system called feedback loop based on the error generated in the work practice system which has been trapped by the mental model design. Feedback loop is generated based on the analysis of warning codes generated by considering the efficiency of employees performing different job activities in the work practice system. All the employees

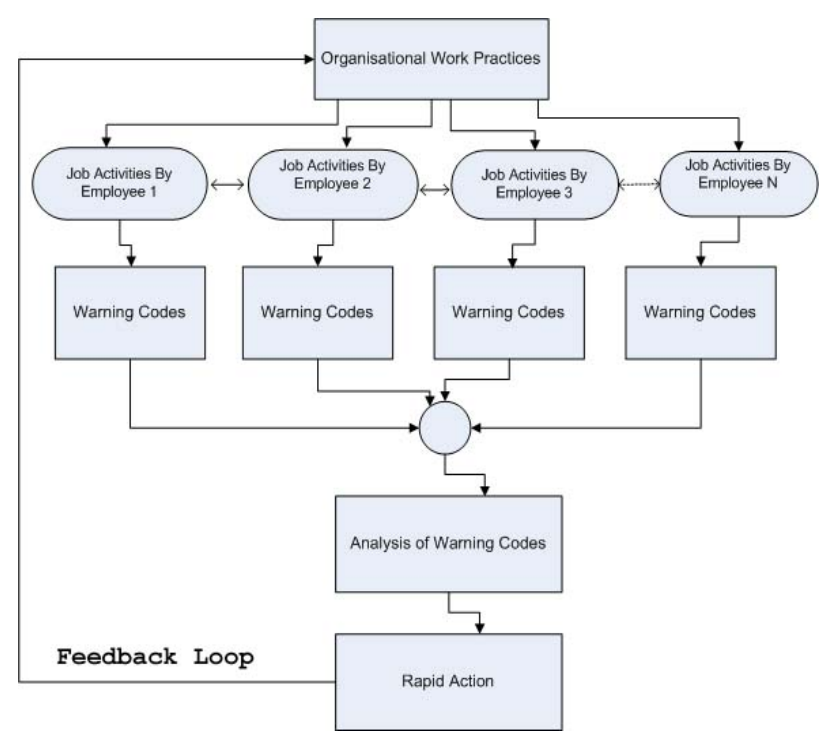

Figure 2. Computer Assisted Alerts based on organisational work practice system. 
are working towards the direction of set goals and targets, it is extremely important for each employee to achieve targets in time to meet the predefined objectives. It has been observed throughout the world, there are many organisations which turned into the failure state and do not come up with positive results, besides, there is no one accepting his mistakes or held responsible providing the reasons for the failure of an organization, therefore, it would be extremely important to trap the errors in time and perform rapid action to save the organisation.

\section{Warning Codes}

Table 1 shows different categories of warning codes based on the error generated in the work practice system. Any rule is violated must be encoded in the form of warning codes and must be reported to the top management which would result in rapid action and support leading to the improvement in the work practice system. Some of the warning codes are serious and need more intense and timely action. This would rescue the whole system in case of failure state. All the warning codes are designed with significance importance provided with ample information which must lead to right decision and timely action. It has been observed from different software applications, warning codes are not designed properly and effectively which may lead us to wrong or weak decision making [1]. Once everything is defined, warning codes are generated based on the situation in the existing work practice system.

\section{Application of Brahms Modeling and Simulation Concept}

Every employee has different scenario and possess different responsibilities in the work practice system, based on his existing job scenario, his job pattern is defined by considering Brahms Modeling and Simulation [2]. It would be a great importance, how an employee is handling his job matters e.g. dealing with agents, objects, artifacts, activity, timing, geography, communication and knowledge etc., [2].

Table 1. Distribution of warning codes in the work practice system.

\begin{tabular}{cccc}
\hline No. & Warning Codes & Objectives & Effective Range \\
\hline 1 & 001 & Rules Violation & Department \\
2 & 002 & Late Reply & Department \\
3 & 003 & Unknown Status & Department \\
4 & 004 & Health Issues & Department \\
5 & 005 & Absence from Duty & Department \\
6 & 006 & Shortage of Skills & Department \\
7 & 007 & Lack of Communication & Department \\
----- & ----- & ------------ & ---------- \\
$\mathrm{n}$ & $\mathrm{N}$ & Weak Decision Making & Department \\
\hline
\end{tabular}

Brahms provides us the deep insight about humanachine interaction system which helps us to understand how human actually interact with colleagues, documents, and machine, communicate and behave, while performing various job activities at certain interval of time [2]. Brahms Process Model comprise of various independent related models which provides us an opportunity to perform modeling job in an easier and efficient way [2]. These independent models are:

\subsection{Agent}

Every work system has different people having different roles in any organisation with respect to different location. Agent model is based on group level hierarchy of the people which are inter-related together on the basis of mutual interest, personal relationships and location [2].

\subsection{Objects}

This model is based on domain objects and artifacts like tools, documents, desks, machines and vehicles in a hierarchic form [2].

\subsection{Activity}

The activity model is related to the activities performed by agents and objects with the passage of time. Activities of agents and objects are always performed at the group or class level [2].

\subsection{Geography}

The geography model is related to the physical location where agents and objects are located like building, rooms and habitats [2].

\subsection{Timing}

This is time when some activity is performed called situation-action rules (workframes). Different activities take different timing for completion. Workframes sometimes interrupted and sometimes resumed based on certain situation, therefore, we consider these frames as situation dependent [2].

\subsection{Knowledge}

This model is based on agent's reasoning which can be represented as forward-chaining production rules called throught frames which come under group and class levels and these thought frames can be inherited [2].

\subsection{Communication:}

The communication model is based on the activity performed by agents and objects through exchanging beliefs and information. A conversation is modeled as an activ- 
ity with the help of communication actions like face to face discussion, by email or by telephone [2].

Real work practice system is keenly observed and human behaviour is measured by deep observation, questionnaire and by interviews. Human behaviour is simulated by comparing the results of real work practice system. Verification and analysis of each and every activity is monitored till the simulation results matches with the real work practice system [2].

Brahms Simulation application provides us the general behaviour of work practice system with respect to time using the concept of various activities, communication and movement of each agent and object in the work practice system. Brahms simulation engine executes the complete model once it is finalised consequently, relational database is created by the simulation engine which involves all the events. Brahms display tool known as Agent Viewer is used to display all the different agents, objects, activities, and areas etc., [2]. We can check the timeline of different activities in the work practice system by using Agent viewer Tool, therefore, it would be easy for us to generate the warning alerts in case of any error caused by the human behavioural activities in the work practice system and rapid action can be taken for the customer service improvement in any organisation.

\section{Conclusions and Future Work}

Quality assurance factor is achieved and customer service has been improved which was a practical challenge for the concerned authorities in any organisation. Human error is unpredictable and it is very hard and complex to judge someone's actual behaviour while performing certain job activities.

It happens specially when we are working as a team in an organisation and whole efficiency is compromised due to some regular human errors in the system at various levels. Human behaviour changes with respect to the mood, time and situation. However, our research study monitors most of the human behavioural activities and diagnoses problematic area, where potential errors are made, consequently, it is checked and well identified in order to achieve the best quality results. Secondly, warning alerts can be designed effectively according to the mental understanding of the people so that timely rapid action can be taken effectively and efficiently.

Future work can be done in the direction of human behaviour studies in more details as it includes various dimensions which needs more investigation and analysis.
Moreover, human behaviour is based on mood, emotions, relationships and sensitivity which cannot be captured one hundred percent, therefore, it is suggested to work in more details about Brahms Simulation and Modeling Technique to define Human-Machine interaction system, which will also result in the improvement of warning alert generation at the time of error caused by human behavioural activity at certain interval.

\section{Acknowledgements}

This research is partially funded by the Malaysian Ministry of Higher Education Grant IIUM/504/RES/G/14/3/ 2/2/ERGS and partly supported by Umm Al-Qura University, Makkah, Kingdom of Saudi Arabia. We would like to thank Professor Asadullah Shah and Associate Professor Mohamad Fauzan Bin Noordin for their useful comments.

\section{REFERENCES}

[1] C. Bravo-Lillo, L. F. Cranor, J. S. Downs and S. Komanduri, "Bridging the Gap in Computer Security Warnings A Mental Model Approach,” IEEE, 2011.

[2] M. Sierhuis and W. J. Clancey,"Modeling and Simulating Work Practice: A Method for Work Systems Design,” IEEE, 2002.

[3] L. JEAN CAMP, "Mental Models of Privacy and Security,” IEEE, Tech \& Society Magazine, FALL 2009.

[4] C. Bravo-Lillo, L. F. Cranor, J. Downs, S. Komanduri and M. Sleeper, "Improving Computer Security," P. Campos et al. (Eds.): INTERACT 2011, Part IV, LNCS 6949, pp. 18-35.

[5] L. F. Cranor, "A Framework for Reasoning About The Human in the Loop,” Carnegie Mellon University.

[6] J. Blythe USC, Jean Camp, Vaibhav Garg,"Targeted Risk Communication for computer security,” IUI11, California, USA, 2011.

[7] J. Blythe, A. Botello, J. Sutton, D. Mazzocco, J. Lin, M. Spraragen and M. Zyda, "Testing Cyber Security with Simulated Human,” 2011.

[8] G. S. Poh, N. N. Abdullah, M. R. Z'aba and M. Ridza Wahiddin, "Reasoning of Collaborative Human Behaviour in Security-Criticial Work Practices: A Framework,” Atlantis Press 2012.

[9] J. Blythe, A. Botello, J. Sutton, D. Mazzocco, J. Lin, M. Spraragen and M. Zyda, "Testing Cyber Security with Simulated Human," Copyright 2011, Association for the Advancement of Artificial Intelligence.

www.aaai.org. 